\title{
Trend of Dengue Viral Infection and Circulating Serotypes in Tripura, North East India
}

\author{
Tapan Majumdar¹, Niladri Sekhar Das², Ayan Modak³, Saikat Majumder \\ ${ }^{1}$ Department of Microbiology, Agartala Government Medical College, Tripura and Principal Investigator, Viral \\ Research and Diagnostic Laboratory, Agartala Govt. Medical College. Agartala, Tripura, India. \\ ${ }^{2}$ Department of Microbiology, Tripura Medical College, Agartala, Tripura, India. ${ }^{3}$ Department of Molecular Virology \\ Laboratory, Rajiv Gandhi Centre for Biotechnology (RGCB), Thiruvananthapuram, Kerala. India. ${ }^{4}$ Department of Viral \\ Research and Diagnostic Laboratory, Agartala Government Medical College, Agartala, Tripura, India.
}

\section{ABSTRACT}

\section{BACKGROUND}

One of the major causes of morbidity and mortality in tropical and subtropical regions is Dengue viral infection. This virus belongs to family flaviviridae comprising of four antigenically distinct serotypes DENV 1 - 4. A small number of studies conducted in North Eastern (NE) Region of India reported Dengue cases in Assam, Meghalaya, Nagaland, Manipur and Arunachal Pradesh. However, no studies have been conducted in the state of Tripura, with regard to pattern of Dengue viral infection and its circulating serotypes. Therefore, this study was undertaken to identify the serotypes circulating in Tripura.

\section{METHODS}

Patients with acute febrile illness were tested for detecting Dengue viral infection by MAC ELISA and / or NS1 detection test at Viral Research and Diagnostic Laboratory (VRDL), of a tertiary care centre in Tripura for a period of 3 years. All NS1 positive samples were further tested for presence of viral RNA by Reverse Transcriptase -PCR (RT - PCR) and serotyping was done using serotype specific primers.

\section{RESULTS}

A total of 2515 acute febrile cases seen over a period of 3 years from 2014 to 2017 was tested for Dengue virus infection by serology. Out of 2515 of cases, 405 cases tested for NS1 antigen, where $10.61 \%$ (43 / 405) was NS1 positive. The remaining 2110 cases were tested for IgM antibody MAC ELISA and $15.68 \%$ (331 / 2110) was MAC ELISA positive. Out of all NS1 antigen positive cases $34.88 \%$ of PCR positive and serotype characterisation showed DENV - 1 was predominant serotype followed by DENV - 2 and DENV - 4 respectively.

\section{CONCLUSIONS}

There is a rising trend of Dengue virus infection in Tripura with circulation of multiple serotypes. Moreover, cocirculation of multiple serotypes is a risk to the emergence of recombinant strains and also heterotypic infection in the near future might lead to development of DHF and DSS. Hence, molecular characterization of circulating serotypes may be helpful in addressing the probabilities of Dengue outbreak and possibilities of complications.

\section{KEY WORDS}

Dengue Serotypes, NS1 Antigen, MAC - ELISA, RT-PCR.

\author{
Corresponding Author: \\ Dr. Tapan Majumdar, \\ Associate Professor, \\ Department of Microbiology, \\ Agartala Govt. Medical College, \\ Tripura, India. \\ E-mail: drtapan1@rediffmail.com
}

DOI: $10.14260 / \mathrm{jemds} / 2020 / 571$

How to Cite This Article:

Majumdar T, Das NS, Modak A, et al. Trend of dengue viral infection and circulating serotypes in Tripura, North East India. J Evolution Med Dent Sci 2020;9(36):26252629, DOI: 10.14260/jemds/2020/571

Submission 06-02-2020,

Peer Review 30-07-2020,

Acceptance 06-08-2020,

Published 07-09-2020.

Copyright (C) 2020 JEMDS. This is an open access article distributed under Creative Commons Attribution License [Attribution 4.0 International (CC BY 4.0)] 


\section{BACKGROUND}

Dengue fever has progressively become one of the most important causes of morbidity and mortality in the tropical and subtropical areas throughout the world over last few decades. ${ }^{1}$ In India, Dengue infection is endemic in almost all the States since past two centuries. Dengue, transmitted by Aedes mosquito, has been known to be benign and selflimiting disease. Dengue Viruses (DENVs) belong to the genus Flavivirus and family Flaviviridae. They have four related, but antigenically distinct serotypes designated as DENV - 1, DENV - 2, DENV - 3 and DENV - 4. Generally, the infections are asymptomatic. In case of symptomatic infection clinical presentations are in the form of Classical Dengue fever or Dengue Haemorrhagic fever (DHF) /Dengue shock syndrome (DSS) which is more common in secondary infections ${ }^{2}$ The severity of the dengue viral infection is influenced by secondary infection with heterologous antibodies or with certain Dengue Viral Serotypes and Genotypes. Urbanization and population explosion combined with changes in climate have aggravated the cases of DF/DHF in India recently.3,4 Additionally, frequent air travel among different states of the country contributed and accelerated the viral spread. ${ }^{5}$ Actual number of dengue cases are under reported and many cases are misclassified though the estimated cases of Dengue are 390 million annually worldwide whereas 3.2 million are form SEAR region. ${ }^{6}$ In the first place, Dengue virus was isolated in 1946 but the first virologically confirmed epidemic was noticed in Kolkata and the eastern coast of India in 1963 to 1964.7 Currently all four DENV serotypes are found circulating in most parts of South east Asia. 8,9

Though Dengue has been more conspicuous in North India since 2000, Southern states also been reporting major dengue outbreaks with Chikungunya. On the other hand, Western and Eastern Indian states like Odisha and Maharashtra reported such several outbreaks. ${ }^{9}, 10,11,12$ Vashna Joshua et al in 2016 showed there is an increasing trend of Dengue cases from 39 districts of 12 states including North East in India.13 North Eastern regional serological survey of 1963 offered an insight in the Lohit district and Darrang district of Arunachal Pradesh and Assam respectively.14,15 During Nineties outbreaks by DENV-2 reported in Assam and Nagaland. ${ }^{15,16}$ In 2012, Dutta et al reported Dengue cases from many States of North East Region.17 Since 2010 NVBDCP started reporting cases of dengue fever from all the states of Northeast including Tripura, though molecular study for serotype detection was not done in Tripura. The character of Dengue serotypes in a geographic location like Tripura necessitates the continuous molecular surveillance about the circulating serotypes. Hence a preliminary study was intended to observe the Circulating Trend of dengue Virus and their serotypes in Tripura, North east India.

\section{METHODS}

Study design was cross sectional study with a duration of 3 yrs (2014 - 2017) at VRDL of a tertiary care centre of Tripura following approval of institutional ethical committee. Approximately $3-5 \mathrm{ml}$ of blood was collected along with detailed clinical history from the suspected cases of acute febrile illness who attended AGMC or directly referred to VRDL from PHCs, Private Clinics, private Hospitals, Nursing Home after taking informed consent.

\section{Serological Diagnosis}

NS1 Ag test (Dengue day 1 test, Mitra and Co Pvt ltd) and MAC ELISA (NIV Pune) was done for the diagnosis of dengue fever from serum sample. IgM capture ELISA was performed If fever duration is $>7$ days by the Kit supplied by NIV and NS1 Ag was detected If fever duration is $<7$ days by a immunochromatography test Kit (Dengue Day 1 test, Mitra \& Co Pvt ltd). All NS1 test result was confirmed by MAC ELISA after 7 days.

\section{Viral RNA Extraction}

NS1 Ag positive samples were selected for extraction of RNA. RNA extraction was carried out according to the product insert provided by the manufacturer QIAGEN, Germany QIAmp Viral RNA mini kit. Briefly, $15 \mu \mathrm{L}$ RNA was extracted using $140 \mu \mathrm{L}$ of serum and stored at $-80{ }^{\circ} \mathrm{C}$ until completion of PCR and Serotyping. RT - PCR performed from the extracted RNA for identification of dengue virus which was followed by nested PCR for serotyping using group and serotype specific primers targeting $\mathrm{C}$ - prM gene. ${ }^{18}$

\section{Reverse Transcription}

$5 \mu \mathrm{L}$ of viral RNA was used for C-DNA synthesis.

\section{1st PCR}

RT-PCR was done using sense and antisense primers from Integrated DNA Technologies (IDT) according to Lanciotti et al, 1992.18 According to the protocol of Super script III one step RT-PCR kit (Invitrogen), amplification of the $5 \mu \mathrm{L} C$-DNA was done in Thermo cycler following Denaturation, Annealing and Extension at $94^{\circ} \mathrm{C}$ for $30 \mathrm{secs}, 50{ }^{\circ} \mathrm{C}$ for $30 \mathrm{secs}$ and $720 \mathrm{C}$ for $1 \mathrm{~min}$ respectively. Finally, further extension of $72{ }^{\circ} \mathrm{C}$ for 10 min continued for 35 cycles.

\section{Semi Nested PCR}

$10 \mu \mathrm{L}$ of first PCR product was utilized for master-mix preparation along with $1 \mu \mathrm{L}$ each primer of 4 serotypes. Same amplification protocol was used in the Thermocycler for 35 cycles like $1^{\text {st }}$ PCR. ${ }^{18}$ The sequence and primer ID used are as follows:

\begin{tabular}{|cc|}
\hline Primer ID & Oligonucleotide Sequence \\
TS1 & CGTCTCAGTGATCCGGGGG \\
TS2 & CGCCACAAGGGCCATGAACAG \\
TS3 & TAACATCATCATGAGACAGAGC \\
TS4 & CTCTGTTGTCTTAAACAAGAGA \\
Sense DEN (Multi) & TCAATATGCTGAAACGCGCGAGAAACCG \\
Anti-Sense (Multi) & TTGCACCAACAGTCAATGTCTTCAGGTTC \\
\hline
\end{tabular}

\section{Detection of Serotypes}

$10 \mu \mathrm{L}$ of Amplicon and $1.5 \%$ Agarose gel was used for the detection of serotypes. A specimen is considered positive for a particular dengue serotype based on the size of amplicon 
(DENV - 1 - 455 bp, DENV-2 - 121 bp, DENV - 3 - 290 bp, DENV $-4-396 \mathrm{bp})$

\section{Statistical Analysis}

Data entry and analysis was performed in computer using SPSS - 20 for windows. Data presented with the help of text, tables and charts. Chi - Square test was used for testing the difference between two or more proportions.

\section{RESULTS}

During the study period, $2515(\mathrm{~N}=2515)$ subjects had undergone tests for NS1 Ag detection and IgM antibody detection by MAC ELISA depending on the duration of fever from 2014 to December 2017 (Table I). Out of 2515 number of febrile cases, NS1 Ag was tested for 405 cases $(\mathrm{N}=405)$ and MAC ELISA for 2110 cases $(\mathrm{N}=2110)$ (Table I). NS1 Ag positive cases were found to be $10.6 \%$ (43 / 405) and MAC ELISA was found to be $15.7 \%$ (331 / 2110). Among $13 \%$ (331 / 2551) of seropositive cases males were more affected 67.06 $\%(222$ / 331) than females $32.93 \%$ (109 / 331) predominant age group being 15 to 50 yrs. (Graph I) with no significant urban and rural distribution. No cases of DHF / DSS were detected clinically in the present study. There was gradual increase in seropositivity rate of dengue infection from $3.8 \%$ to $14.5 \%$ during 2014 to 2017 (Table I). However, during the month from September to November the positivity rate (55\%) was much higher (Graph II). All NS1 Ag positive sample were tested for confirmation of dengue Virus by RT - PCR and 34.88 $\%$ (15 / 43) were found positive (Table I).

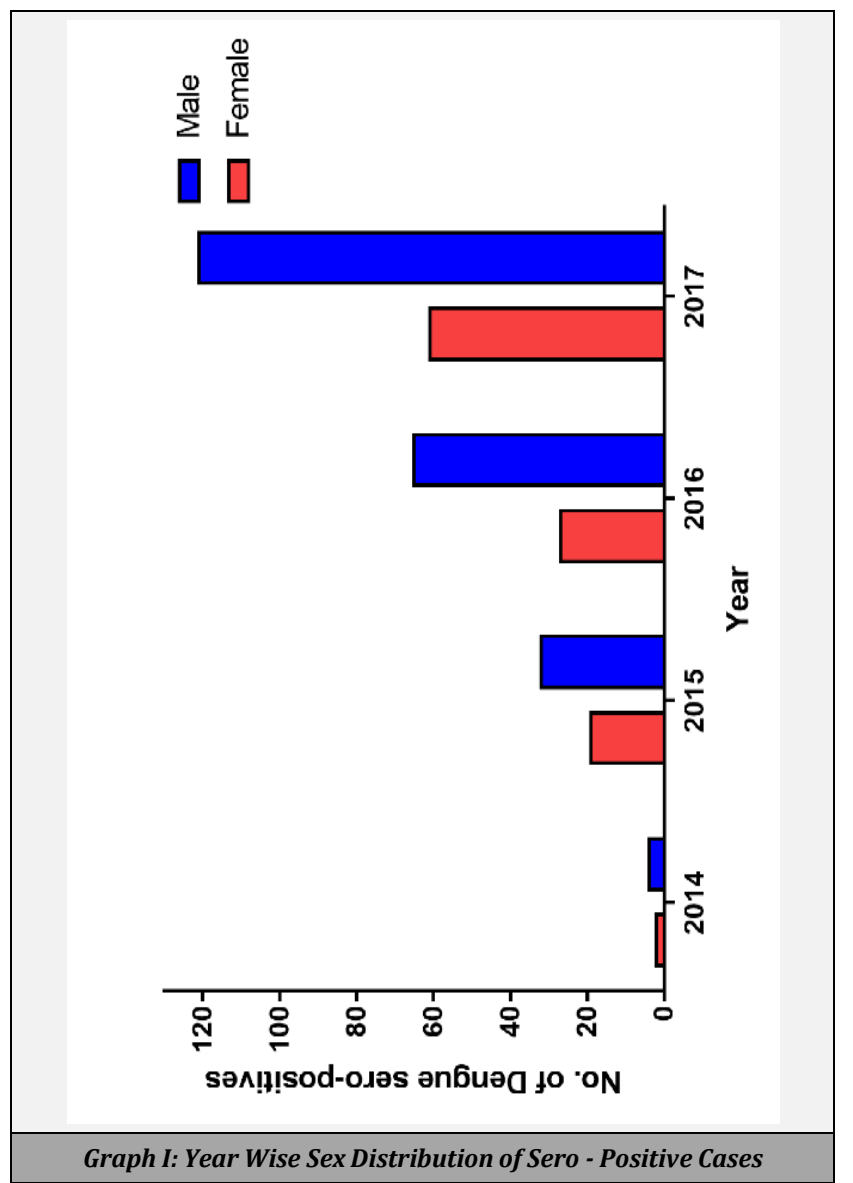

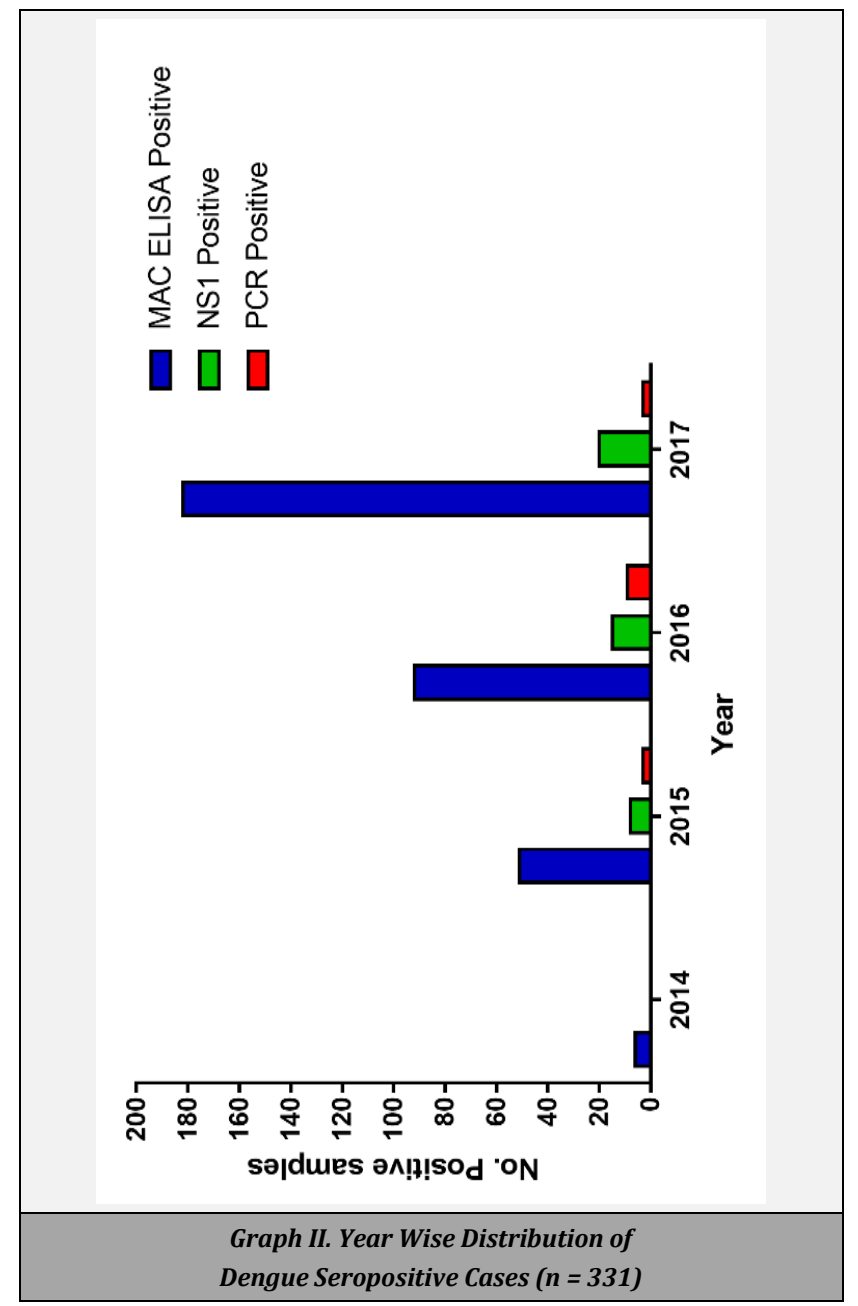

芒

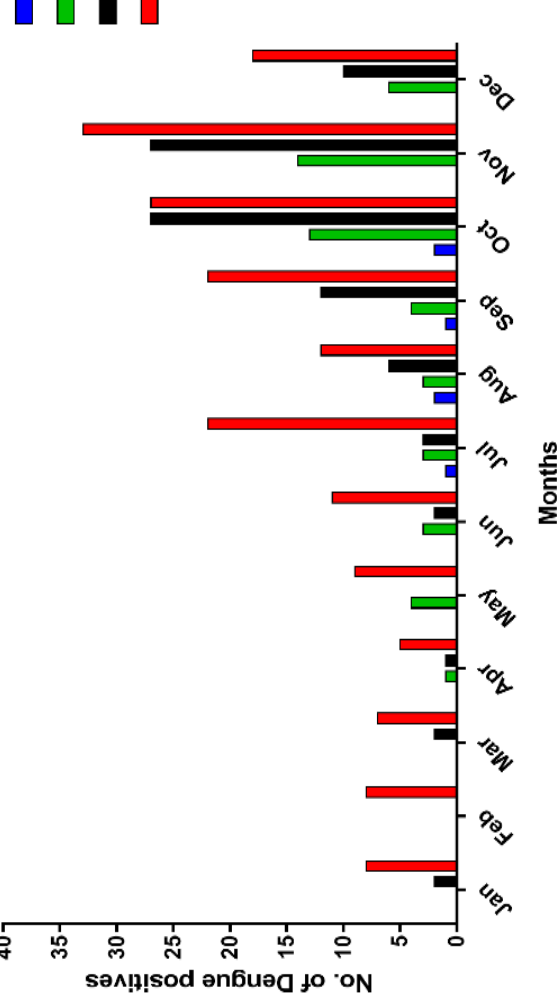

Graph III: Month Wise Distribution Positive Cases 
Serotyping was done among PCR Positive samples using 4 different primers. Common circulating viral Serotypes were found to be DENV - 1, DENV - 2 and DENV-4 (Table 1). Among 15 PCR positive samples $46.67 \%$ was DENV - 1 followed by $33.33 \%$ DENV - 2 and $20 \%$ of DENV - 4. Figure 1 is showing the image of RT-PCR which consist of different serotypes of Dengue.

\begin{tabular}{|c|c|c|c|c|c|}
\hline \multirow[t]{2}{*}{ Year } & \multirow[t]{2}{*}{ Samples } & \multicolumn{2}{|c|}{$\begin{array}{c}\text { Positive Serology } \\
\text { Results }\end{array}$} & \multicolumn{2}{|c|}{$\begin{array}{c}\text { Distribution of } \\
\text { Serotypes }(n=15)\end{array}$} \\
\hline & & NS1 Ag & IgM & & \\
\hline 2014 & 157 & - & 6 & & \\
\hline \multirow{4}{*}{2015} & \multirow{4}{*}{417} & \multirow{4}{*}{8} & \multirow{4}{*}{51} & DEN 1 & 2 \\
\hline & & & & DEN 2 & 1 \\
\hline & & & & DEN 3 & 0 \\
\hline & & & & DEN 4 & 0 \\
\hline \multirow{4}{*}{2016} & \multirow{4}{*}{689} & \multirow{4}{*}{15} & \multirow{4}{*}{92} & DEN 1 & 4 \\
\hline & & & & DEN 2 & 0 \\
\hline & & & & DEN 3 & 2 \\
\hline & & & & DEN 4 & 3 \\
\hline \multirow{4}{*}{2017} & \multirow{4}{*}{1252} & \multirow{4}{*}{20} & \multirow{4}{*}{182} & DEN 1 & 1 \\
\hline & & & & DEN 2 & 2 \\
\hline & & & & DEN 3 & 0 \\
\hline & & & & DEN 4 & 0 \\
\hline Total & 2515 & 43 & 331 & & 15 \\
\hline \multicolumn{6}{|c|}{ All NS1 Ag positive samples were also positive by IgM ELISA, RT-PCR positive $=15$} \\
\hline Table I & est Result A & alysis of & $\log y a$ & RT - PCR & Wise) \\
\hline
\end{tabular}

\section{DISCUSSION}

This study on trend of Dengue viral infection in Tripura highlighted a steady increase in sero-positivity from $3.8 \%$ to $14.5 \%$ during the study period of $2014-2017$. Patient age is a risk factor for severe dengue disease and age shift clearly indicates an epidemiological change in Dengue infection. The present study demonstrated more number of affected males than females predominantly in the age group of $15-50$ yrs. More numbers of Dengue infected Males were reported in various scientific reports. ${ }^{19,20}$ Male preponderance may be due to preferential health seeking behaviour in male dominating society. Ecological imbalance due to rapid urbanisation, changes in lifestyle and socio-economic changes may lead to spread of dengue virus from urban to rural areas.21 Though this study showed no significant variation between Urban and rural population regarding Dengue infection, cases were detected from all the 8 districts of Tripura. Seasonal variation was observed in the present study. This may be due to high vector density during monsoon and post monsoon months mainly September to November. ${ }^{22,23}$ A Delhi based study correlated the association of rainfall, temperature, humidity with the distribution of seropositive cases which increased at the end of monsoon during October and November. ${ }^{24}$ Only $34.88 \%$ of NS1 positive cases were positive by PCR. This may be due to late detection after several days of illness minimizing the sensitivity of PCR, as NS1 Ag remains in circulation for longer period. This study showed mean duration for PCR positive samples are 2.1 days. The epidemiology of dengue virus and its prevalent serotypes keeps on changing. From public health importance point of view, DENV infection pattern monitoring is most essential. Using serotype specific primers, we found 3 circulating serotypes DENV - 1, DENV - 2 and DENV - 4. Major circulating serotype was found to be DENV-1 (46.7\%) followed by DENV 2 and DENV 4. Co circulation of multiple serotypes may result in coexisting infection in some patients with more than one serotype. ${ }^{23}$ The
Occurrence of co-infection with multiple DENV serotypes possesses the risk of emergence of recombinant strains.25 There has been a significant increase in cases almost every consecutive year with spread to places where it was not reported in the past, thereby making Dengue a major public health problem in many parts of India. DF and DHF outbreaks have been occurring since 1824 and multiple epidemics took place during the year 1836, 1906, 1911, 1972, 2005 that affected $40 \%$ of the city people in West Bengal, which is a Dengue endemic zone. ${ }^{26}$ Though Dengue is hypo endemic in North Eastern States of India despite the fact that it is surrounded by several dengue endemic countries like Myanmar, Bangladesh, Thailand and Indonesia. Serological evidence has been reported, that suggests the occurrence of DENV-2 activity in Assam and Nagaland in early 90s. In 2008, there was a major outbreak of dengue in Manipur.16,23 During 2006 DF epidemic $30 \%$ of the reported cases of outbreak was due to DENV 1.23 DENV - 2 came to the fore as the primary serotype from the early 1970 s to 2000 when it brought about the large epidemics of DF in 1933 and DF / DHF in 1996.27,28 In Tripura Stereotyping was done for the first time, resulting in detection of three circulating serotypes DENV1, 2, 4. Co circulation of multiple serotype might lead to heterotypic infection in subsequent years. This will increase the chances of development of DHF and DSS in our State.

\section{CONCLUSIONS}

With the increasing trend of Dengue infections in last three years, detection of circulating serotypes and their genotypes by molecular methods will be helpful in predicting the probability of Dengue outbreak in the near future. The present study highlights the importance of molecular characterisation of DENVs, as the circulating serotypes usually gets replaced with a new serotype which may bear the potential of causing severe outbreak.

As the study was carried out in a tertiary care set up, the present data may not be extrapolated for the whole state which is a limitation of the study. Continuing surveillance of acute febrile illness is needed throughout the year mainly in the month of September to November to generate more robust epidemiological data of circulating Dengue viruses in our state. This will immensely help in timely intervention and taking appropriate public health measures.

The authors are grateful to Directorate of Health Research (DHR), India for the financial support and grants. Authors thank the staff of VRDL, AGMC for the technical help.

\section{REFERENCES}

[1] Monath TP. Dengue in the arboviruses. In: Monath TP, ed. Ecology and epidemiology. Boca, Raton, USA: CRC Press 1988:224-60.

[2] Nimmannitya S. Dengue and dengue haemorrhagic fever. In: Cook GC, Zumla AI, eds. Manson's tropical diseases. $21^{\text {st }}$ edn. Philadelphia, USA: Saunders 2003: p. 7672. 
[3] Gupta N, Srivastava S, Jain A, et al. Dengue in India. Indian J Med Res 2012;136(3):373-90.

[4] Raheel U, Faheem M, Riaz MN, et al. Dengue fever in the Indian subcontinent: an overview. J Infect Dev Ctries 2011;5(4):239-47.

[5] Monath TP. Dengue: the risk to developed and developing countries. Proc Natl Acad Sci USA 1994;91(7):2395-400.

[6] World Health Organisation 1948, https://www.who.int/en/news-room/factsheets/detail/dengue-and-severe-dengue accessed on 1 October, 2018.

[7] Ramakrishnan SP, Gelfand HM, Bose PN, et al. The epidemic of acute haemorrhagic fever, Calcutta 1963: epidemiological Inquiry. Indian J Med Res 1964;52:63350.

[8] Murray NEA, Quam MB, Wilder-Smith A, et al. Epidemiology of dengue: past, present and future prospects. Clin Epidemiol 2013;5:299-309.

[9] Anoop M, Issac A, Mathew $T$, et al. Genetic characterization of dengue virus serotypes causing concurrent infection in an outbreak in Ernakulam, Kerala, South India. Indian J Exp Biol 2010;48(8):849-57.

[10] Ceellia D, Kakade AB, Bhagat MB, et al. Detection of dengue -4 virus in Pune, Western India after an absence of 30 years -its association with two sever cases. Virol J 2011;8:46.

[11] National Vector Borne Disease Control Programme (NVBDCP) 2003, India, Dengue cases and deaths in the country since 2008. https://www.nvbdcp.gov.in/dencd.html

[12] Das B, Sahu A, Das M, et al. Molecular investigations of dengue virus during outbreaks in Orissa state, Eastern India from 2010 to 2011. Infection Genetics Evolution 2013;16:401-10.

[13] Joshua V, Murhekar MV, Ashok M, et al. Mapping dengue cases through a national network of laboratories, 20142015. Indian J Med Res 2016;144(6):938-41.

[14] Rodrigues FM, Dandawate CN. Arthropod-borne viruses in north eastern India: a serological survey of Arunachal Pradesh and northern Assam. Indian J Med Res 1977;65(4):463-5.

[15] Dutta P, Mahanta J. Potential vectors of dengue and the profile of dengue in the north-eastern region of India: an epidemiological perspective. Dengue Bulletin 2006;30:234-42.

[16] Sankari T, Hoti SL, Singh TB, et al. Outbreak of dengue virus serotype-2 (DENV-2) of Cambodian origin in Manipur, India - association with meteorological factors. Indian J Med Res 2012;136(4):649-55.

[17] Dutta P, Khan SA, Borah J, et al. Demographic and clinical features of patients with Dengue in Northeastern region of India: a retrospective cross sectional study during 2009-2011. Journal of Virology and Microbiology 201;2012:1-11.

[18] Lanciotti RS, Calisher C, Gubler DJ, et al. Rapid detection and typing of dengue viruses from clinical samples by using reverse transcriptase polymerase chain Reaction. J Clin Microbiol 1992;30(3):545-51.

[19] Pandey A, Diddi K, Dar L, et al. The evolution of dengue over a decade in Delhi, India. J Clin Virol 2007;40(1):87-8.

[20] Sinha N, Gupta N, Jhamb R, et al. The 2006 dengue outbreak in Delhi. India. J Commun Dis 2008;40(4):243-8.

[21] Guha-Sapir D, Schimmer B. Dengue fever: new paradigms for a changing epidemiology. Emerg Themes Epidemiol 2005;2(1):1.

[22] Pandey N, Nagar R, Gupta S, et al. Trend of dengue virus infection at Lucknow, North India (2008-2010): a hospital based study. Indian J Med Res 2012;136(5):862-7.

[23] Chakravarti A, Arora R, Luxemburger C. 50 years of dengue in India. Trans $\mathrm{R}$ Soc Trop Med Hyg 2012;106(5):273-82.

[24] Chakravarti A, Kumaria R. Eco-epeidemiological analysis of dengue infection during an outbreak of dengue fever, India. Virol J 2005;2:32.

[25] Worobey M, Rambaut A, Holomes EC. Widespread intraserotype recombination in natural populations of dengue virus. Proc natl Acad Sci USA 1999;96(13):7352-7.

[26] Hati AK. Studies on dengue and dengue hemorrhagic fever (DHF) in West Bengal state, India. J Communi Dis 2006;38(2):124-9.

[27] Bharaj P, Chahar HS, Pandey A, et al. Concurrent infections by all four dengue virus serotypes during an outbreak of dengue in 2006 in Delhi, India. Virol J 2008;5:1.

[28] Kumar A, Sharma SK, Padbidri VS, et al. An outbreak of dengue fever in rural areas of northern India. J Commun Dis 2001;33(4):274-81. 